\title{
Squeeze Film Damping of Doubly Supported Ribbons in Noble Gas Atmospheres
}

\author{
C. S. Gudeman, B. Staker, and M. Daneman \\ Silicon Light Machines \\ 385 Moffett Park Drive, Suite 115 \\ Sunnyvale, CA 94089-1208
}

\begin{abstract}
Doubly supported MEMS ribbons of a Grating Light Valve device have been characterized for damping time when excited by an impulse in noble gas atmospheres. A simple linear relationship is found between the damping time of the ribbons and the gas viscosity when corrected for rarefaction effects.

\section{INTRODUCTION}

The Grating Light Valve (GLV ${ }^{\mathrm{TM}}$ ) modulator is an emerging technology employing doubly supported beams (ribbons) as elements of a projection display array [1]. The low effective mass and high tension in these ribbons permit operation at speeds of up to $20 \mathrm{MHz}$, which is the highest operational speed of all MEMS ribbons or cantilevers to date. The geometry and speed of the GLV ribbons are such that viscous forces in atmospheric gas phase environments play a vital role in damping the mechanical response of ribbons when actuated by an electrical impulse. To understand this viscous damping a detailed study of GLV ribbon ring down characteristics has been made in inert atmospheres of $\mathrm{He}, \mathrm{Ne}, \mathrm{Ar}$, $\mathrm{Kr}$, and $\mathrm{N}_{2}$ at $1 \mathrm{~atm}$ pressure and in $\mathrm{N}_{2}$ at 0.5 and $1.5 \mathrm{~atm}$. The results are reported in this article. From these data we analytically relate damping time to the viscosity and Knudsen Number of the gas and the geometry of the ribbon. In the current case solution of the Reynolds Equation is unnecessary. A simple closed form expression of the gas viscosity, corrected for slip flow according to Burgdorfer [2], is sufficient to accurately predict the effect of gas viscosity for all gases and pressures studied.
\end{abstract}

\section{GRATING LIGHT VALVE TECHNOLOGY}

Measurements of MEMS ribbons reported here are based on the grating light valve structure, which is depicted in Fig. 1. The device is built up of thin films and processes that are common in the fabrication of CMOS electronic devices, with the major difference being that, like most MEMS devices, a layer of sacrificial material is required for the release of the device from the underlying layers. For the GLV structure this sacrificial layer is polycrystalline silicon that is isotropically etched out from under the ribbons. The thickness of this sacrificial layer determines the air gap spacing $y_{0}$ that, as will be seen below, is the nominal thickness of the gas squeeze film.

Referring to Fig. 1, the ribbon arrays are built up on silicon substrates. The substrate itself is used as the bottom electrode. The sacrificial layer is deposited, followed by an etch step to create post vias for ribbon support and electrical connections. The $\mathrm{Si}_{3} \mathrm{~N}_{4}$ ribbon layer of nominally $100 \mathrm{~nm}$ thickness is deposited, followed by the top aluminum electrode layer. The ribbons are patterned, thus exposing a path to the sacrificial layer for the release etch, which is the final step. The dimensions of the ribbon can be varied over a very broad range. Experimentally lengths of 15 . $300 \mu \mathrm{m}$ and widths of $2.0-7.75 \mu \mathrm{m}$ have been fabricated, and have shown predicted resonant frequencies and actuation voltages. The air gap, defined by the sacrificial layer, has been varied from $150 \mathrm{~nm}$ to $750 \mathrm{~nm}$, again yielding predictable behavior in the GLV dynamics.

The GLV device is ideal for the study of ribbon dynamics, since the defracted light of the GLV grating provides a means of sensing the ribbon deflection. Because it is the diffraction of light that is used in this study to sense the dynamic response of the ribbon, a brief description of GLV diffraction will be presented next.

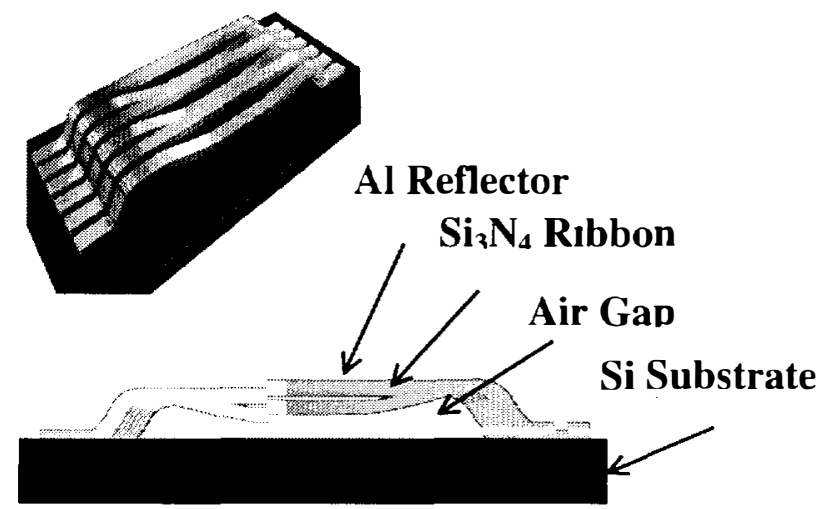

Figure 1. Diagram of the GLV structure. Upper left relief drawing depicts a pixel in the bright state.

Consider normal incidence illumination of the device with collimated monochromatic light (cf. Fig. 2). The light will be reflected back to the source as shown in Fig. 2a when the ribbons lie in a single plane. This is the case when no potential difference is applied between the ribbon aluminum and the bottom electrode. If a non-zero potential is applied to alternate ribbons in the array (Fig. 2b), these ribbons will be electrostatically attracted to the bottom electrode, causing them to deflect. A phase grating is created, and light is diffracted at angles $\theta$, where

$$
\theta=\arcsin (m \mathcal{N} d)
$$


Here $\Lambda$ is the wavelength of light, $d$ is the period between deflected ribbons, and $\mathrm{m}$ is a signed integer representing the order of diffraction. Note that the defraction angle is independent of the ribbon deflection $\delta y$. For the current case first order light is diffracted at roughly four degrees. The intensity of light that is diffracted into first order does vary with the magnitude of the deflection, with the following dependence:

$$
I=I_{s} \sin ^{2}(2 \pi \delta y / \Lambda)
$$

Here $I_{0}$ is the maximum intensity defracted into $1^{\text {st }}$ order. Thus the ribbon response to an applied voltage impulse can be sensed as a function of time from the intensity of light diffracted into first order. $\delta y(t)$ can then be determined from the ratio $\mathrm{I} / \mathrm{l}_{\mathrm{o}}$, given the wavelength of incident light $\Lambda$.

\section{Dark State}

Incident light reflected
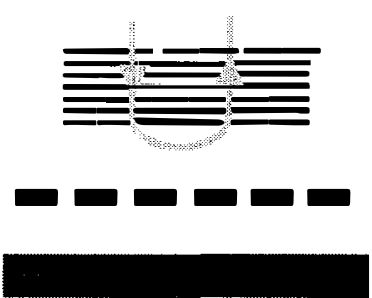

\section{Bright State} Incident light

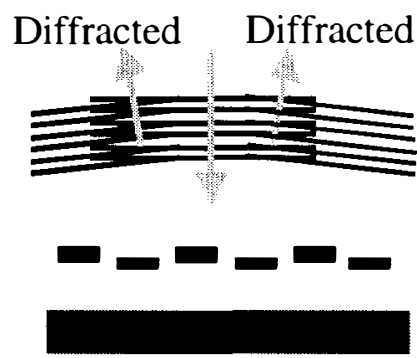

Figure 2. Diagram of the GLV pixel operation. The dark state occurs when the all six ribbons are coplanar and light is specularly reflected back to the source. The bright state occurs when alternate ribbons are deflected a distance of $\mathrm{N} 4$ and the light is diffracted.

\section{EXPERIMENTAL}

The diffracted light from a damped device is monitored in an environmental chamber as shown in Fig. 3. The light from a 680 $\mathrm{nm}$ diode laser is directed through a large (6 inch diameter) window in a vacuum chamber onto the ribbon array with an incidence angle of 30 degrees and a focus of $25 \mu \mathrm{m}$. In the undeflected state the ribbons reflect the incident light specularly back though the window. When the ribbons are deflected, light is lost from of the specular beam and transferred into the diffraction orders, which also pass back through the chamber window. The $1^{\text {st }}$ order beams are the most intense and are well separated from the specular beam. A photodetector is placed in the path of one of the $1^{\text {st }}$ order lobes, and the output from the photodetector is displayed on a digitizing oscilloscope and finally captured by a computer. Noise in the system is dominated by laser noise in the $50 \mathrm{MHz}$ passband of the photodetector.

The impulse applied to the GLV aluminum layer is a 100 $\mathrm{kHz}$ square wave that is offset to be unipolar with an adjustable amplitude of $10-30 \mathrm{~V}$. The rise and fall times of the impulse are approximately $100 \mathrm{nsec}$. The amplitude is adjusted to deflect the ribbons roughly $50 \%$ of $\Lambda_{e f f} / 4$ or $\sim 100 \mathrm{~nm}$. (Note that here $\Lambda_{e f}=$ $\Lambda / \cos \theta$, where $\theta=$ angle of incidence with respect to the normal. This factor permits the simple treatment above to be applied to the more general case of non-normal incidence.) This provides large intensity modulation (as can be inferred from Eq. 2), but avoids close approach to the bottom electrode, where complications of small separations (snap down and the surface roughness contribution to the effective air gap separation) arise.

Prior to a series of ring down measurements, consisting of ring down curve acquisition of both rising and falling impulse edges for a range of ribbon arrays spanning $70-100 \mu \mathrm{m}$ in length, the vacuum chamber is evacuated to a base pressure of $\sim 10^{-6}$ Torr. The chamber is then back filled with the chosen gas to the desired pressure (generally 1 atmosphere), as measured by a Bourdon gauge. The measurements are made in a static gas system, and the level of outgassing contamination during the $\sim 30$ minute measurement series is less than $100 \mathrm{ppm}$. The gases studied were $\mathrm{He}, \mathrm{Ne}, \mathrm{Ar}, \mathrm{Kr}$, and $\mathrm{N}_{2}$ at 1 atm. Additionally ring down data is reported here in $\mathrm{N}_{2}$ at 0.5 and $1.5 \mathrm{~atm}$.

\section{DATA ANALYSIS}

Fig. 4 shows the $1^{\text {st }}$ order optical response following both rising and falling edges of an applied impulse. Note that the rising edge of the impulse induces a ringing response characteristic of a simple damped harmonic oscillator. The optical response following the falling edge, on the other hand, appears doubled in frequency and the intensity for each cycle returns to a common

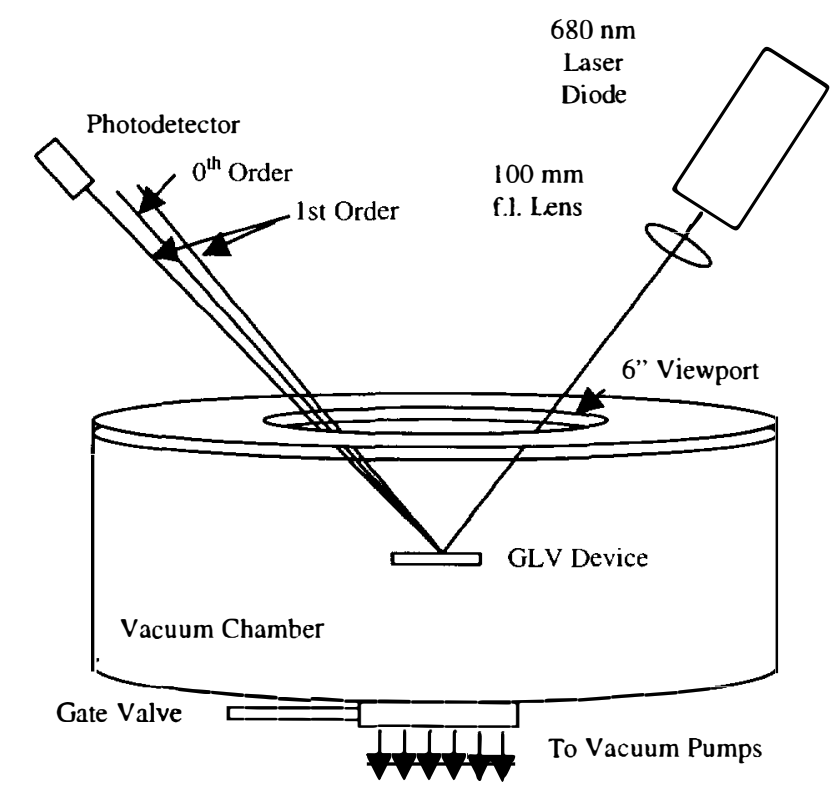

Figure 3. Diagram of the test apparatus, including the environmental chamber optics probe.

base line. This "rectification" following the falling edge occurs because the ribbons ring about the undeflected plane, where the optical response function is even, as inspection of Eq. 2 shows when $\delta y$ is nominally zero. Following the rising edge the ribbons 
ring about a steady state deflection of $\sim 100 \mathrm{~nm}$, where the optical response function is approximately linear.

Although the rising and falling edges have seemingly different character, a single function describing both has fairly simple form:

$$
I=D+I_{v} \sin ^{2}(2 \pi / \Lambda)\left(B+A e^{-t / \tau} \sin (2 \pi v t+\phi),\right.
$$

where $\delta y$ in Eq. 2 has been replaced by $B+A e^{-\nu \tau} \sin (2 \pi v t+\phi)$. This expression provides terms for the steady state position $B$ of the ribbons following the impulse edge, the undamped ringing amplitude $A$, exponential damping with time constant $\tau$, harmonic oscillation at frequency $v$, and a phase factor $\phi$. The additive constant $D$ is included to accommodate the photodetector dark current and all other DC offset contributions of the detection system.

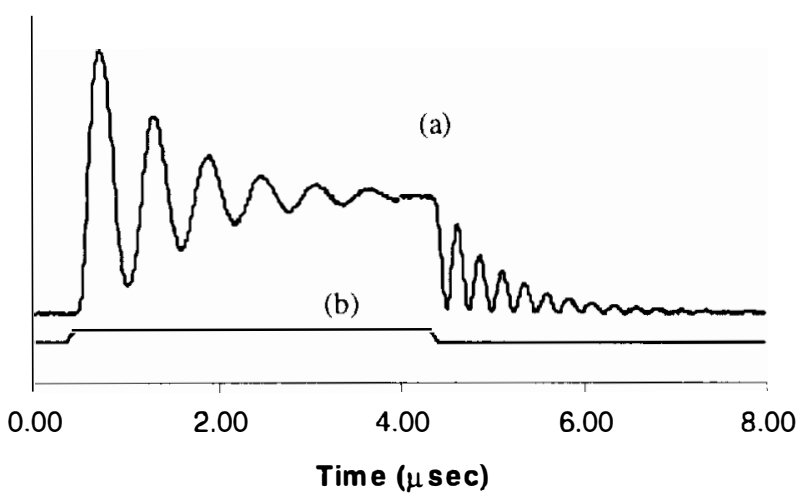

Figure 4. Optical response of a GLV pixel (a) following impulse excitation (b) of the ribbons in He gas at $1 \mathrm{~atm}$.

A typical ring down data set, such as the one shown in Fig. 4 , consists of 1000 points sampled at a rate of $125-250 \mathrm{MHz}$. This intensity vs. time data set is then fitted to Eq. 3 using the Levenberg - Marquardt nonlinear least squares algorithm [3] Prior to fitting, the constants $D$ and $I_{o}$ are determined by direct measurement of the dark response at zero applied ribbon voltage and the peak response occurring when the ribbons are deflected by $\Lambda / 4$. The fit then determines the parameters $A, B, \nu, \tau$, and $\phi$. Generally convergence occurs in $15-25$ iterations, requiring $\sim 10$ seconds of computation time on a $133 \mathrm{MHz}$ P5 computer in either of two interpreted languages (APL2PC for DOS and LABVIEW 4.0). Stable convergence is particularly sensitive to the initial guess of the phase factor $\phi$. Thus it is convenient to code the fit such that initial guess of $\phi$ is easily adjustable. Residuals (not shown here to avoid congestion in the plot) are typically $<5 \%$ of the peak signal response.

The responses to rising and falling edges must be fitted separately since all 5 fitting parameters are in general different in the two states. A brief qualitative description of these differences for $v$ and $\tau$ is germane to this analysis, since the ringing behavior in the deflected state (following the rising edge) is encumbered by two factors that make it a less accurate measure of viscous effects than that of the undeflected state. Briefly, these differences can be described as follows. First, spring softening, an effective reduction in the spring constant and therefore the natural frequency of the ribbon, occurs because the mechanical restoring force $-k_{\text {mech }} \delta y$ is opposite in direction to the electrostatic force $k_{\text {elec }} V^{2} /\left(y_{0}-\delta y\right)^{2}$ that deflects the ribbon. Here $V$ is the deflecting potential and the $k$ 's are positive proportionality constants. Thus in all cases, the natural frequency $v$ is observed to be lower in the deflected state. Second, in the deflected state the ribbon takes on a half-sine profile, and thus on average the air gap is non-uniform along the length of the ribbon, with the air gap at the center of the ribbon considerably less than the nominal value $y_{o^{*}}$ As a result, the damping time $\tau$ is experimentally observed to be lower in the deflected state. Because of these effects, only ringing behavior data from the undeflected state is reported in this article.

\section{RESULTS}

Fig. 5 shows extracted values of $v$ and $\tau$ for all ribbon lengths studied. In all five of these cases in this figure, the gas pressure is $1 \mathrm{~atm}$. Several observations are evident.

First, $v$ shows little dependence on damping gas, but falls off with inverse ribbon length, as expected. Conversely, $\tau$ shows a very marked dependence on damping gas, but is essentially independent of ribbon length. It is the dependence of $\tau$ on damping gas that is examined next.

Table 1 summarizes several properties of the damping gases including viscosity $\eta$ and mean free path $\lambda$ at the specified pressures for ambient temperature $(297 \mathrm{~K})$. Note that although $\mathrm{Ne}$

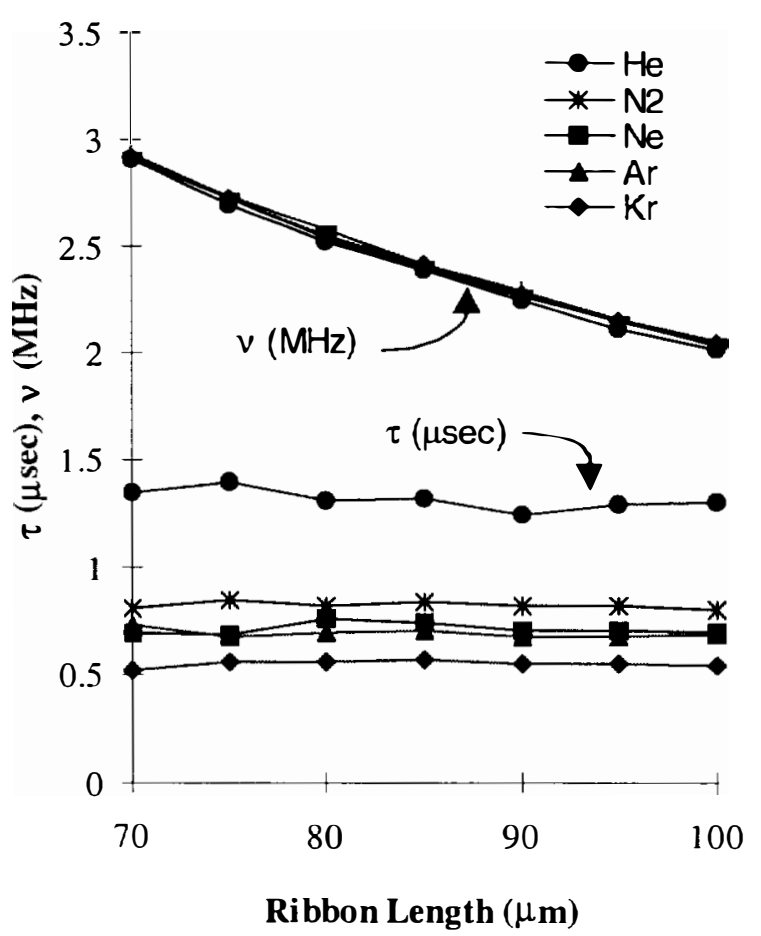

Figure 5. Values of natural frequency $v$ and damping time $\tau$ extracted from fits of GLV optical response in the undeflected state. Note that the curves for $v$ fall nearly on top of one another.

possesses the highest viscosity of the series, $\mathrm{Kr}$ provides the most 
efficient damping. Thus viscosity alone is insufficient to accurately describe the damping behavior. For the case reported here, where the nominal air gap $(650 \mathrm{~nm})$ is comparable in length to the mean free path $\lambda$ of these gases, rarefaction effects occur, even at atmospheric pressure. Veijola, et al [4]. have summarized the various correction factors to gaseous viscosity accounting for rarefaction in the gas. In general these corrections vary with the Knudsen number $K_{n}=\lambda / y$. For low $K_{n}(<1)$ the various correction factors generally converge to the expression derived by Burgdorfer [2]:

$$
\eta_{\text {eff }}=\eta /\left(1+6 K_{n}\right)
$$

The effective viscosity values thus calculated are also given in Table 1 , where $\mathrm{y}$ is assumed to be $650 \mathrm{~nm}$ and $\lambda$ is calculated using

$$
\lambda=\frac{1}{\sqrt{2} \pi d_{o}^{2} n},
$$

a result of the simple kinetic theory of gases [5].

If it is now assumed that $\tau$ is inversely proportional to $\eta_{\text {eff }}$, this simple correction factor can easily be tested. Fig. 5 shows a plot of $\tau$ vs. $\left(\eta_{\text {eff }}\right)^{-1}$ for the five noble gases at 1 atm. Additionally, this plot includes data for $\mathrm{N}_{2}$ damping at 0.5 and 1.5 atm pressure. The $\mathrm{R}^{2}$ correlation value for a straight line of 0.995 indicates the effectiveness of this correction factor.

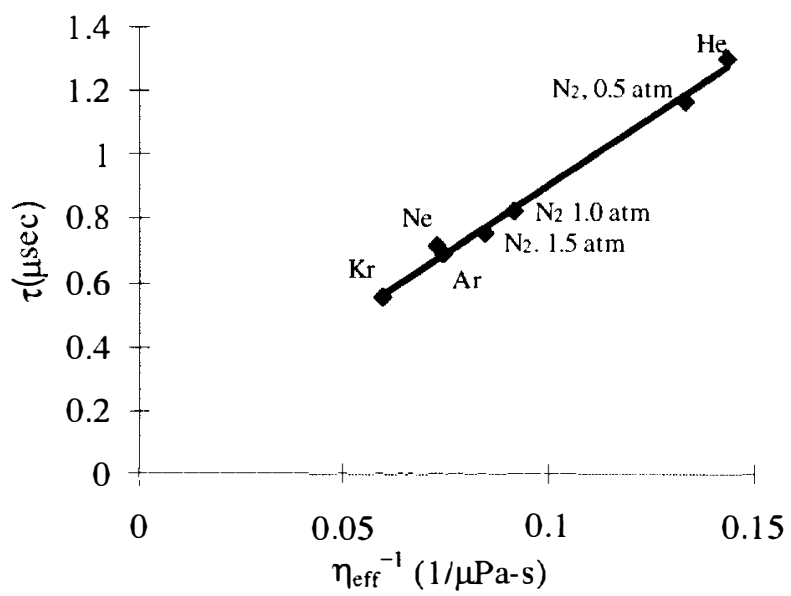

Figure 6. Damping time vs. the effective viscosity of the damping atmosphere at 1 atm pressure (unless otherwise noted).

Further testing of this model can be made by again assuming $\tau=$ $k \eta_{\text {eti }}$ and rearrangement Eq. 4, yielding

$$
\tau \eta=k+(6 k \lambda / y)
$$

A plot of $\tau \eta$ vs. $\lambda$ then gives a line with slope of $6 \mathrm{k} / \mathrm{y}$ and intercept k. Note that $y$ in this case is the nominal air gap $y_{0}$, yielding $y_{0}=$
$624 \mathrm{~nm}$. This is in very good agreement with the process target of $650 \mathrm{~nm}$.

Table 1. Summary of viscosity, mean free path ( $\mathrm{nm}$ ), effective viscosity, and damping time at $20^{\circ} \mathrm{C}$ for the gas atmospheres used in this study.

\begin{tabular}{c|cccc}
\hline Gas & $\begin{array}{c}\text { Viscosity }^{a} \\
(\mu P a-s)\end{array}$ & $\begin{array}{c}\text { Mean free } \\
\text { path }^{b} \\
(\mathrm{~nm})\end{array}$ & $\begin{array}{c}\text { Effective } \\
\text { Viscosity } \\
(\mu P a-s)\end{array}$ & $\begin{array}{c}\tau \\
(\mu \mathrm{sec})\end{array}$ \\
\hline $\mathrm{He}$ & 19.41 & 194 & 6.96 & 1.3 \\
$\mathrm{Ne}$ & 31.11 & 138 & 13.73 & 0.71 \\
$\mathrm{~N}_{2}$ & 17.49 & 67 & 10.91 & 0.82 \\
$\mathrm{Ar}$ & 22.17 & 70 & 13.51 & 0.69 \\
$\mathrm{Kr}$ & 25.04 & 53 & 16.80 & 0.55 \\
$\mathrm{~N}_{2}(0.5 \mathrm{~atm})$ & 17.49 & 134 & 7.51 & 1.16 \\
$\mathrm{~N}_{2}(1.5 \mathrm{~atm})$ & 17.49 & 44 & 11.82 & 0.75 \\
\hline Ref.6 & \multicolumn{4}{c}{}
\end{tabular}

a) Ref. 6.

b) From Eq. (5).

\section{CONCLUSIONS}

A simple slip flow model that accurately describes the squeeze film damping of doubly supported MEMS ribbons when actuated by an electrical impulse has been demonstrated. The viscosity of five different gases was shown to be correctable for rarefaction effects in the damping gas by a scaling factor that depends only on the Knudsen number of the gas in the air gap under the ribbon.

\section{REFERENCES}

1. R. Apte, F. Sandejas, W. Banyai, and D. Bloom, "Grating Light Valves for High Resolution Displays," Solid State Sensors and Actuators Workshop, June 1994. D. Bloom, "The Grating Light Valve: Revolutionizing Display Technology," Projection Displays III Symposium, SPIE Proceedings Volume 3013, February 1997.

2. A. Burgdorfer, "The Influence of the Molecular Mean Free Path on the Performance of Hydrodynamic Gas Lubricated Bearings," J. Basic Eng., ASME, 81 (1959) 94-99.

3. W. H Press, B. P. Flannery, S. A. Teukolsky, and W. T. Vetterling, Numerical Recipes in C, Cambridge Press, Cambridge, 1988.

4. T. Veijola, H. Kuisma, J. Lahdenpera, and T. Ryhanen, "Equivalent-circuit Model of the Squeezed Gas Film in a Silicon Accelerometer," Sensors and Actuators A, 48 (1995) 239-248.

5. J. F. O'Hanlon, A User's Guide to Vacuum Technology, John Wiley and Sons, New York, 1980.

6. Handbook of Chemistry and Physics, $51^{\text {st }}$ edition. Ed. Robert C. Weast, TheChemical Rubber Company, Cleveland, OH (1971) 